\title{
Prevalence of MSDs among Nurses: A Study in Indian Health Care Industry
}

\section{Singh LP*}

Department of Industrial and production Engineering, Dr. B. R. Ambedkar NIT, India

*Corresponding author: Lakhwinder Pal Singh, Department of Industrial and production Engineering, Dr. B. R. Ambedkar NIT, Jalandhar 144011, India, Email: singhl@nitj.ac.in

\section{Research Article}

Volume 2 Issue 3

Received Date: May 07, 2018

Published Date: May 22, 2018

DOI: $10.23880 /$ eoij-16000149

\section{Abstract}

Nursing is the largest, the most diverse, and one of the most respected of all the health care professions. The demand for nursing remains high, and projections suggest that such demand will substantively increase. Awkward posture, lifting, forceful movements and manual work at rapid rate contribute to musculoskeletal disorders in nurses. Currently, India is facing a shortage of more than million nurses and this scenario needs to be rectified.

Objective: Present study is focused on assessing risk of musculoskeletal disorders (MSDs) among nurses engaged in different activities of healthcare.

Method: A video film was recorded in real postures during the various actives performed by the nurses. Snaps were cropped after a fixed interval for each of the activity. The work postures were evaluated using REBA tool.

Results: As per the REBA assessment, 3\% nurses required immediately corrective measures and 20\% nurses needed to correct their postures as soon as possible, $52 \%$ nurses needed further investigation due to moderate risk, $22 \%$ nurses are at low risk and only 3\% are having negligible risk. Furthermore using REBA tool and questionnaires, it is found that major contributor in this MSDs of nurses is the back. 36\% nurses reported problem in their back due to forward bending most of the time, $10 \%$ are having problem in their neck, $26 \%$ nurses reported problem in feet due to standing and breaks of lesser duration. This paper is restricted to only posture analysis on observation based, however application of ergonomics posture was suggested to the nurses.

Conclusion: Awkward postures and manual patient handing in the workplace should be reduced. There is strong need of ergonomics techniques and low cost automation, in form of movable beds, elevators, application of various sensors to reduce the need of continuous monitoring, so that workload can be reduced and efficiency can be increased to affect the organizational cost and the society as a whole

Keywords: MSD; Nurses; Indian Health Care Industry; Stress; Musculoskeletal Disorders 


\section{Ergonomics International Journal}

\section{Introduction and Literature}

Ergonomics is a human factor with the application of scientific information concerning objects, systems and environment for human use. The field of ergonomics is an applied science that also studies the effects of fatigue and discomfort on the body and designs protective equipment to keep operators safe and efficient. Specifically, physical ergonomics deals with the human body's responses to physiological stress. There is an amazing lack of awareness and knowledge about this field even today. It is the professional and ethical duty of all designers to keep ergonomics above all other considerations, and ensure that the interaction between their design and its final user does not become unpleasant, difficult to use (difficult to reach and clean too), confusing, tiring, illegible, unintelligible, mistake-prone, harmful or dangerous even in the smallest possible way (Gupta, 2004). Ergonomics (or human factors) is the scientific discipline concerned with the understanding of interactions among humans and other elements of a system, and the profession that applies theory, principles, data and methods to design in order to optimize human well-being and overall system performance [1]. Erdinc \& Vayvay reported that ergonomics interventions that enhance employee performance lead to better quality in manufacturing.

Ergonomics is the theoretical and fundamental understanding of human behavior and performance in purposeful interacting sociotechnical systems, and the application of that understanding to design of interactions in the context of real systems. Balasubramanian, et al. provided a single measure of the ergonomic assessment in terms of several factors (e.g. posture, biomechanical forces, environmental, etc.) collectively contributing to ergonomic impairments. This integrated score provides a clearer picture of the risk involved in the job and hence it can be used as a basis for prioritizing operations for ergonomic interventions. This article refers to the systems and scientific knowledge applied to lifting, turning and guiding heavy objects, namely patients.

Patient handling and movement tasks are physically demanding, generally performed under unfavorable conditions and often unpredictable in nature. Patients offer multiple challenges including variations in size, physical disabilities, cognitive function, level of cooperation and fluctuations in condition. As a given weight to be lifted, they are awkward packages, lack even weight distribution and have been known to become combative during the lift process. Nursing has one of the highest occupational injury rates of any profession. Data from the Bureau of Labor Statistics (BLS) show that in
2011, the rate of overexertion injuries averaged across all industries was 38 per 10,000 full time workers. By comparison, the overexertion injury rate for hospital workers was twice the average $(76$ per 10,000$)$, the rate for nursing home workers was over three times the average $(132$ per 10,000$)$, and the rate for ambulance workers was over six times the average $(238$ per 10,000$)$ [2]. The single greatest risk factor for overexertion injuries in healthcare workers is the manual lifting, moving and repositioning of patients, residents or clients, i.e., manual patient handling. Most of the patient handling which occurs in health care settings is performed by nurses and support staff such as nursing aides and orderlies. The most recent data available from the BLS (2010) show that within the health care industry, workers in these occupations suffered the most lost-time cases of general musculoskeletal pain $(11,960)$ and back pain $(4,700)$ [3]. Nurses and other bedside healthcare nurses are at greater risk for sustaining musculoskeletal disorders than most nurses. For comparison, consider that truck drivers ranked second and nurse eighth. In India, some of the issues related to nurse retention still remain to be tackled-lack of technological advancement, poor ergonomics considerations in the government and private sectors, lack of a conducive work environment and infrastructure facilities. Twelve percent of nurses nationwide leave the profession annually because of back injuries and more than 52 percent of nurses complain of chronic back pain [4]. The lifting problem has become so detrimental to bedside healthcare personnel that many private hospitals, nursing homes, and state and government healthcare agencies have set forth no-lift policies and procedures in an attempt to drastically cut the number of injuries, time away from work, initial and chronic pain, need for pain alleviation treatment, and loss of staff. Occupational safety and health programs have fostered research to identify injury risk factors and safety interventions to prevent injuries during patient handling. Evidence-based research has shown that safe patient handling interventions can significantly reduce overexertion injuries by replacing manual patient handling with safer methods guided by the principles of Ergonomics [5].

The objective of this study is to analyze the working postures of nurses engaged in various processes of health industry. The study used REBA (Rapid Entire Body Assessment) assessment tool and recommended the changes to be made in the body posture while working. REBA (Rapid Entire Body Assessment) was developed by Hignett $S$ and Mc Atamney L to provide a quick and easy observational postural analysis tool for whole body activities (static and dynamic giving musculoskeletal risk 


\section{Ergonomics International Journal}

action level. The development of REBA is aimed to divide the body into segments to be coded individually with reference to movement planes. It provides a scoring system for muscle activity caused by static, dynamic, rapid changing or unstable postures. It reflects that coupling is important in handling of the loads but may not always be via the hands. It also gives an action level with an indication of urgency.

Manual patient handling is one of the major causes of severe injury. There are multiple reasons why thousands of nursing injuries occur each year. Slip and slide injuries are related to everything from a leaky urine collection bag to improper signage following the damp cleaning of floors. Even the nursing shortage is being blamed for many forms of nurse injuries. The fourth most commonly reported injury during work is injury related to poor ergonomics. Simply stated, nurses and bedside healthcare nurses are moving patients in unsafe conditions because a second pair of hands or helpful lifting equipment is just not available. In sharp contrast are lifting techniques adhered to by physical and occupational therapists, regardless of a dearth of willing and able hands and bodies for patient movement and transfers. Deborah $\mathrm{X}$ Brown, herself a casualty of an ergonomic disaster, believes there are no ergonomically safe ways to lift patients and indicates experts have known this for more than 15 years [6]. Nurses hurt not only themselves, but also the patients in their charge. Hoisting patients under their arms or bending and twisting to place a patient in a wheelchair from the bed leads to dislocations, shearing injuries, musculoskeletal and nerve damage, and the opportunity for internal injuries as well. Following our ergonomically savvy European colleagues, adopting a nolift policy in your facility can reduce lift and strain injuries across the population who lifts people as part of a daily occurrence.

A research on Ergonomic Interventions for Reducing Musculoskeletal Disorders documented a combination of physical, psychological, and psychophysical workplace risk factors in most industrialized countries. Risk factors for the development of low back pain include (moderately) flexed, laterally bent or twisted trunk postures, high forces on the hands, high one time or accumulated forces on the spine, and vibration [7]. Similar workplace physical risk factors are associated with high levels of musculoskeletal disorders in the neck, shoulders and arms. Physical risk factors such as high forces, high repetition, working with arms overhead, long term static postures, local contact forces and vibration are commonly identified. It includes perceptions of low control and poor workplace social environment. Other risk factor includes uncomfortable static position, contact stress of muscles and tendon and also extreme temperature condition [8]. This study enhanced the awareness of the risk factors which may occur in the construction industry. The Strain Index to Analyze Jobs for Risk of Distal Upper Extremity Disorders was developed. Three different studies have shown that it is capable of identifying jobs with no distal upper extremity morbidity as "safe" and jobs with distal upper morbidity as "hazardous."

\section{Materials and Methods}

The following steps were followed as a part of the methodology followed in undertaking this project:

- The method adopted for posture analysis was REBA (Rapid Entire Body Assessment) as it allowed for the assessment of the entire body which could not be done with either RULA (Rapid Upper Limb Assessment) or NIOSH Lift Equation.There is no need of special equipment for this posture analysis using REBA. There are standard charts for these methods, the individual only has to study \& analyse the postures using videos and photos, then quantify the fatigue using charts and suggest the remedial action based on the risk factor obtained of their current working posture.

- A questionnaire was prepared for qualitative analysis of postures considering various prospects like age, work experience, working hours, common areas of problem, overtime hours and many such important factors. The data was collected from various hospitals of Jalandhar and Ludhiana cities, so as to get a brief idea about the overall work culture of nurses. This data was collected via questionnaires, videos and interaction with the staff.

- Video films (about 90) were recorded on nurses when they were attending patients and performing different activities giving emphasis over the each activity. After recording the video, the snapshots were cropped after a fixed amount of time gap. The snapshots of 487 in numbers were cropped in such a way that a range of postures of body parts in the activities were clear to observe. The snapshots were analyzed to fill the scores in REBA score sheets (Figure 1). The jobs with the involvement of high risk were numbered higher and those with less risk involvement were numbered 1 . Immediate corrective actions and necessary changes were recommended for activities numbered higher to avoid any risk. In REBA the posture is assessed by dividing body parts into sections and each body part is scored according to its range of movement. Higher scores are given to the body parts where presence of risk factors are more and lower scores are given to those where presence of risk factors are minimal. The REBA scores were divided into 


\section{Ergonomics International Journal}

five categories: negligible, low, medium, high and very high. Medium, high and very high needed an immediate action to avoid any musculoskeletal disorders (Table 1).
- Overall Score was calculated using REBA scoring. This score is the value of Risk Factor and accordingly remedial action was suggested.

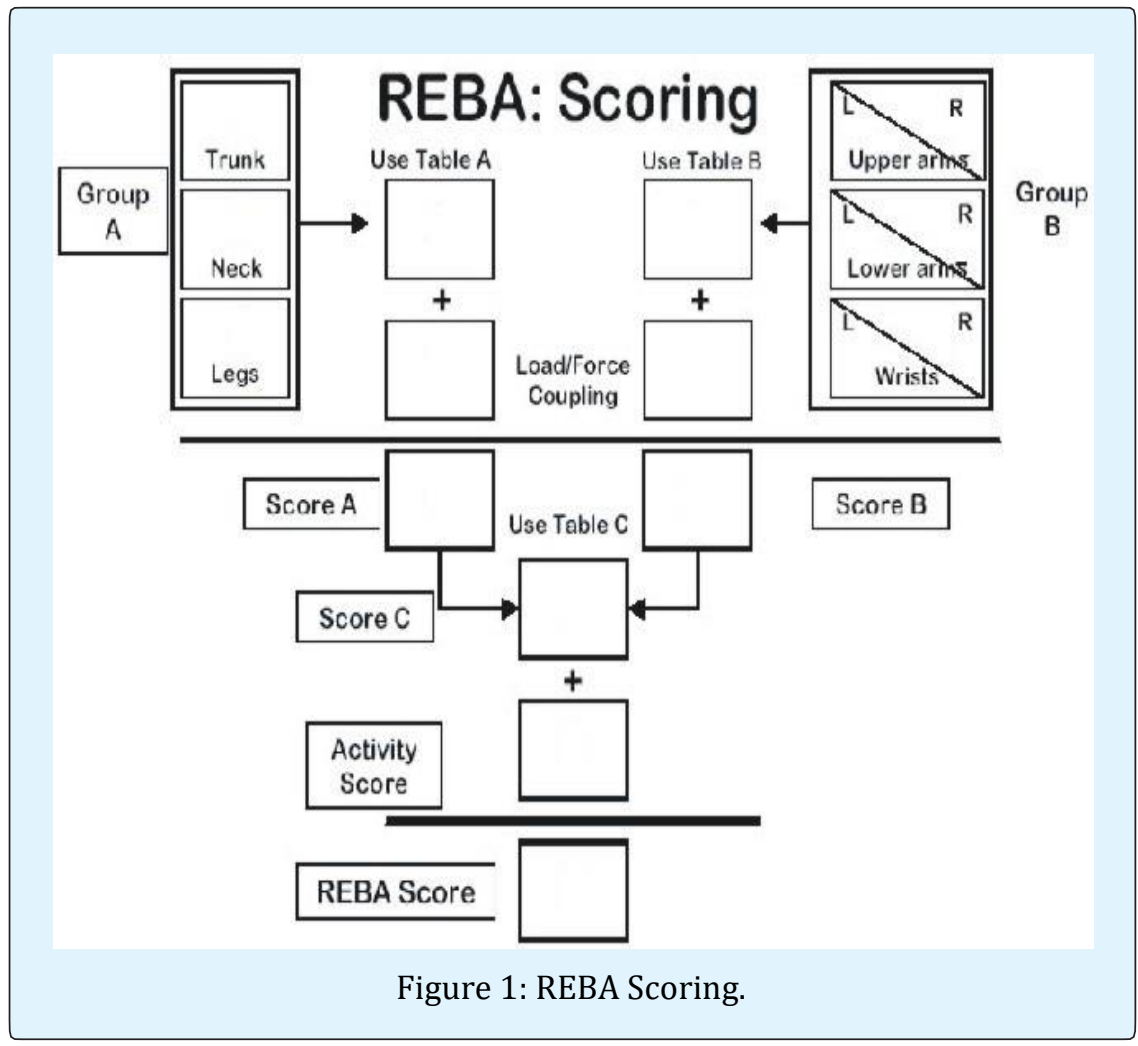

\begin{tabular}{|c|c|c|c|}
\hline $\begin{array}{c}\text { Action } \\
\text { level }\end{array}$ & $\begin{array}{c}\text { REBA } \\
\text { score }\end{array}$ & Risk level & $\begin{array}{c}\text { Action (including } \\
\text { further assessment) }\end{array}$ \\
\hline 0 & 1 & Negligible & None necessary \\
\hline 1 & $2-3$ & Low & May be necessary \\
\hline 2 & $4-7$ & Medium & Necessary \\
\hline 3 & $8-0$ & High & Necessary soon \\
\hline 4 & $11-15$ & Very high & Necessary NOW \\
\hline
\end{tabular}

Table 1: REBA Action Levels.

\section{Results and Discussion}

The data obtained from the questionnaires was scored. $45 \%$ of the nurses surveyed were married and $55 \%$ of the nurses surveyed were unmarried. About $72 \%$ of the nurses belong to the age group of 20-30 years and only $13 \%$ are of the age 30 and above 30 . It was observed that there was no significant difference between the amounts of break time that nurses from most age group take. Although as the age keeps on increasing, it was found that nurses experienced fatigue sooner as compared to younger age groups. Nurses above the age of 35 experience more problems in knees $(16 \%)$ as compared to younger age groups where the percentage of nurses experiencing problem in knees is $8 \%$ on an average. Nurses above the age of 35 also complain more of problems in shoulders as compared to nurses from other age groups.

The percentage (number) distribution of nurses with different frequency of Symptoms is shown in Table 2, it reveals that $2.86 \%$ reported that they always experienced problem in their back, $40.48 \%$ reported that they often experienced problems in their back while $42.38 \%$ reported that that they experienced pain in their back sometimes. Only $7.62 \%$ and $6.67 \%$ reported that they seldom or never experienced problems with their back. The frequency with which problem was experienced were given scores on a scale of 0 to 4 corresponding to never and always. The weighted mean of these values was calculated and thus the rating was calculated. It turned out to be 2.8. The rating is a direct indication of the risk of disorder due to that area. $0.95 \%$ reported that they always experienced problem in their feet, $24.76 \%$ reported that they often experienced problems in their back while $26.19 \%$ reposted that that they experienced 


\section{Ergonomics International Journal}

pan in their feet sometimes. $28.57 \%$ and $19.52 \%$ reported that they seldom or never experienced problems with their feet. The frequency with which problem was experienced were given scores on a scale of 0 to 4 corresponding to never and always.

The weighted mean of these values was calculated and thus the rating was calculated. It turned out to be 1.59 . $0.48 \%$ reported that they always experienced problem in their neck, $2.86 \%$ reported that they often experienced problems in their neck while $3.33 \%$ reposted that that they experienced pan in their neck sometimes. $42.86 \%$ and $50.48 \%$ reported that they seldom or never experienced problems with their neck. The frequency with which problem was experienced were given scores on a scale of 0 to 4 corresponding to never and always. The weighted mean of these values was calculated and thus the rating was calculated. It was found to be 0.6 , which shows that there is less risk of injury in this area. $7.62 \%$ reported that they always experienced problem while bending, $29.52 \%$ reported that they often experienced problems while bending, while $52.38 \%$ reposted that that they experienced pain while bending sometimes. Only $6.19 \%$ and $4.29 \%$ reported that they seldom or never experienced problems during bending.
The frequency with which problem was experienced were given scores on a scale of 0 to 4 corresponding to never and always. The weighted mean of these values was calculated and thus the rating was calculated. It turned out to be 2.3 on a scale of $0-4.30 .95 \%$ reported that they always experienced problem while standing, $44.76 \%$ reported that they often experienced problems while standing. $15.71 \%$ reported that that they experienced pain while standing sometimes. Only $2.38 \%$ and $6.19 \%$ reported that they seldom or never experienced problems while standing. The frequency with which problem was experienced were given scores on a scale of 0 to 4 corresponding to never and always. The weighted mean of these values was calculated and thus the rating was calculated. It was found to be 2.92 on a scale of $0-4$.

According to the data obtained via questionnaires and interaction with the staff of the hospitals, nurses are walking or are in standing position most of the time $30 \%$ each), followed by bending posture which constitutes for about $24 \%$ of the total time. The subjects spent least time in sitting posture and lifting loads, which was $9 \%$ and $7 \%$ respectively the same is exhibited in Figure 2.

\begin{tabular}{|c|c|c|c|c|c|c|c|}
\hline Symptoms in & Total & Always & Often & Sometimes & Seldom & Never & Scale(0-4) \\
\hline Back & 210 & $6(2.86)$ & $85(40.48)$ & $89(42.38)$ & $16(7.62)$ & $14(6.67)$ & 2.38 \\
\hline Feet & 210 & $2(0.95)$ & $52(24.76)$ & $55(26.19)$ & $60(28.57)$ & $41(19.52)$ & 1.59 \\
\hline Neck & 210 & $1(0.48)$ & $6(2.86)$ & $7(3.33)$ & $90(42.86)$ & $106(50.48)$ & 0.6 \\
\hline Bending & 210 & $16(7.62)$ & $62(29.52)$ & $110(52.38)$ & $13(6.19)$ & $9(4.28)$ & 2.3 \\
\hline Standing & 210 & $65(30.95)$ & $94(44.76)$ & $33(15.72)$ & $5(2.38)$ & $13(6.19)$ & 2.92 \\
\hline
\end{tabular}

Table 2: Percentage (number) distribution of nurses with different frequency of Symptoms.

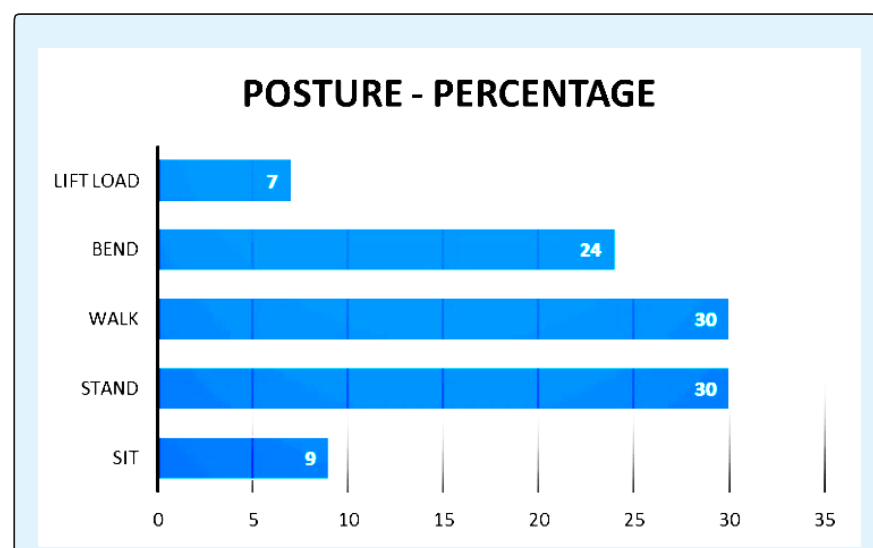

Figure 2: Distribution of Time in Different Postures.
It is shown in Figure 3 that most nurses reported problems in the back which constituted for about $36 \%$ of the total nurses surveyed. The next biggest area of problem was feet as reported by $26 \%$ of the total nurses surveyed. $10 \%$ of the nurses surveyed experienced problems in their necks. Only $8 \%$ of the total nurses reported problems in their knees followed by $8 \%$ of the nurses suffered problems in their shoulders as a result of incorrect work postures. Areas which were found to be less problematic were wrists and elbows with $7 \%$ and $5 \%$ reported problems respectively. 


\section{PROBLEMS IN BODY PARTS - PERCENTAGE}

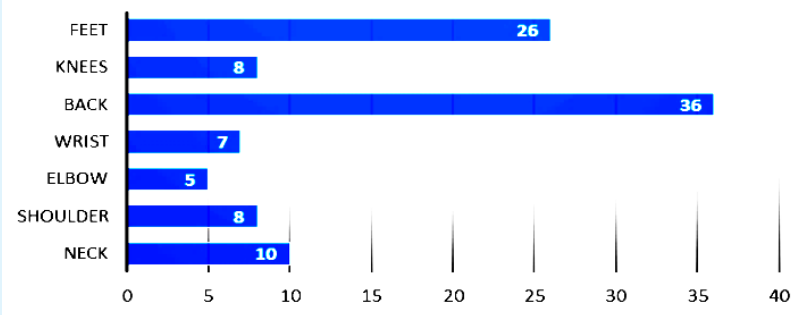

Figure 3: Distribution of MSDs in different body parts.

Videos were paused after a fixed interval and the postures of the nurses were scored according to REBA assessment sheet and their risk factors were accordingly calculated is shown in Figure 4. It is observed that 3\% of the nurses were at very high risk of any musculoskeletal disorder. In ICU, while changing drips their upper arm is raised and stretched beyond the normal working range. As well as they frequently bend and stretch their trunk for drip. Thus immediate action was suggested in their case. Whereas $20 \%$ of the nurses were at high risk of MSDs. During the forward bending of nurses, to do medication of patients they have to twist their trunk and neck. Thus, corrective measures in their work postures were soon required. Majority i.e. 52\% of the nurses were found to be at medium risk of MSDs due to their working posture. Trunk \& neck are the main contributors to this risk factor. As they have to check patient mostly in the forward bending posture and they have to stand for a longer duration. Therefore, changes in their work postures were suggested to be implemented soon.

Around $22 \%$ of the nurses were at low risk of injuries. Thus, it was suggested that corrective measures may be required in their case. Merely 3\% of the nurses were found to be at zero or negligible risk of injuries. This shows that a very low percentage of nurses are working safely. However, rest of the $97 \%$ is under the risk of MSDs. That is a very peculiar situation, the underlying reasons include; tight work schedule i.e. in this profession is such that nurses don't get break and are busy all the time. Due to overuse of their body especially low back, feet/legs, they are suffering from overuse syndromes. Nurses working engaged in operation theatres experience fatigue sooner than other nurses of the same age group and work load on account of the environment inside the operation theatre. Moreover, the operation theatre requires the nurses to remain active as their performance needs to be optimum all the time. The harmful gases inside the theatre often cause their fingers to turn dark at the tips and some parts of their limbs to get numb. Further, the chances to have contracted with some diseases are enhanced. Thus, on account of the data obtained, it can be concluded that most of the nurses have reduced efficiencies and short careers as a result of incorrect work postures during their work hours. The Body-Mass Index of the nurses was calculated and they were accordingly categorized. The analysis of the data thus obtained reveals that the work schedule of the nurses is such that they are bound to experience fatigue and problems in feet and back. The BMI of theses nurses is of very less significance when it comes to the causes of such MSDs in nurses. To add to this, the nurses have to be at their feet all the time to take care of the emergency cases that may walk in at any point of the day. They need to look after the patients after fixed intervals of time irrespective of the amount of fatigue or pain that they may be experiencing.

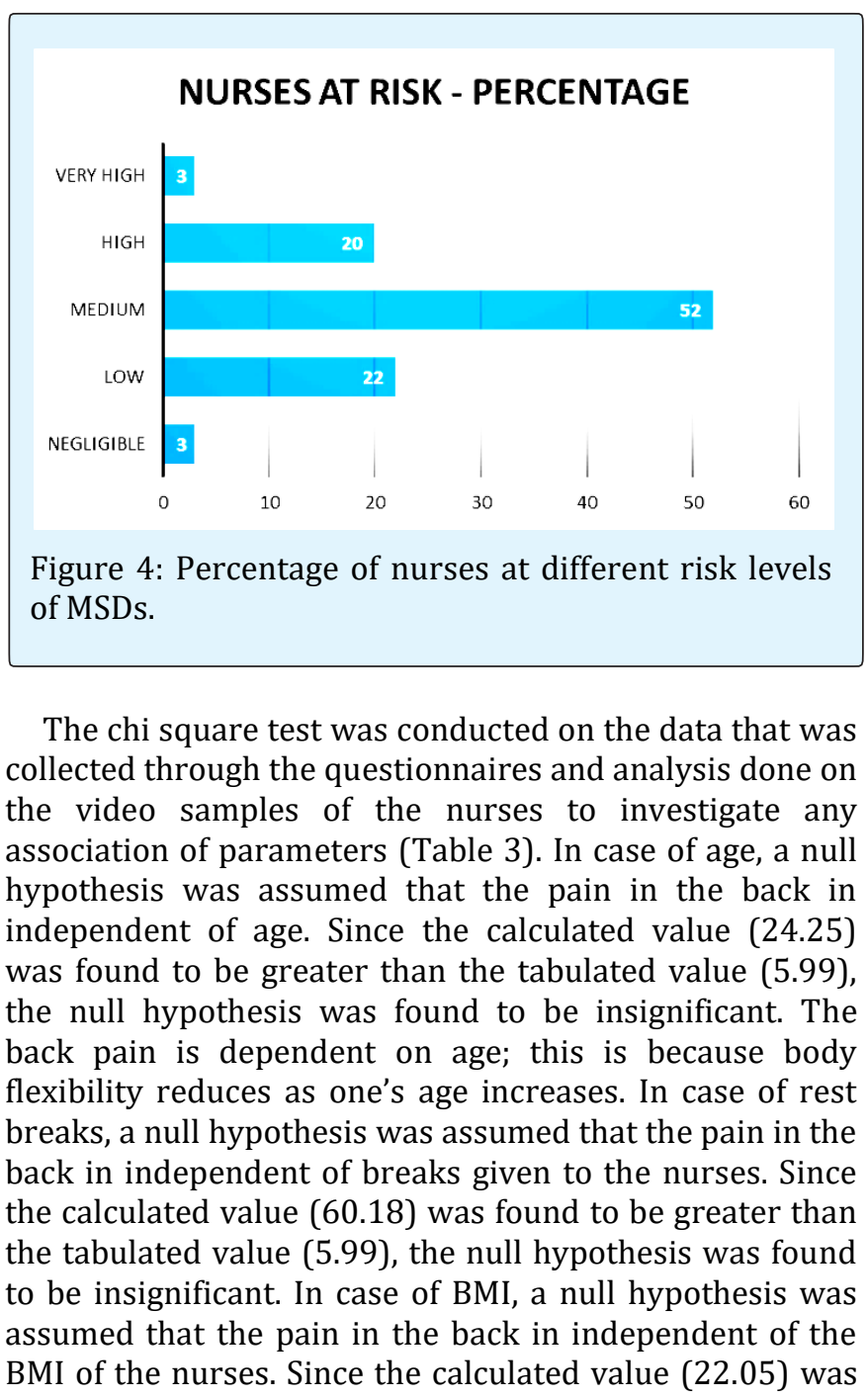


found to be greater than the tabulated value (5.99), the null hypothesis was found to be insignificant. In case of postures, a null hypothesis was assumed that the pain in the back in independent of postures of the nurses. Since the calculated value (9.62) was found to be less than the tabulated value (4.77), the null hypothesis was found to be insignificant. The risk of disorders in nurses is highly dependent on the work postures of the nurses. The REBA score of the nurses under high and very high risk of disorders was cross checked with the data obtained from their questionnaires. It was concluded that the major contributor to injuries in back and the main posture in which problem is experienced is bending [9-14] (Figure 5).

\begin{tabular}{|c|c|c|c|c|}
\hline & $\mathbf{f}_{\mathrm{o}}$ & $\mathbf{f}_{\mathrm{e}}$ & $\boldsymbol{x}^{2}$ & calculated value \\
\hline \multirow{6}{*}{ Age } & 10 & 25.89 & 9.74 & \multirow{6}{*}{24.25} \\
\hline & 93 & 68.14 & 9.06 & \\
\hline & 67 & 73.29 & 0.54 & \\
\hline & 22 & 32.23 & 3.25 & \\
\hline & 3 & 6.19 & 1.64 & \\
\hline & 1 & 0.42 & 0.82 & \\
\hline \multirow{5}{*}{ Posture } & 10 & 6 & 2.67 & \multirow{5}{*}{9.62} \\
\hline & 4 & 6 & 0.67 & \\
\hline & 31 & 20 & 6.05 & \\
\hline & 87 & 90 & 0.1 & \\
\hline & 62 & 65 & 0.14 & \\
\hline \multirow{5}{*}{ Break } & 28 & 28.72 & 0.01 & \multirow{5}{*}{60.18} \\
\hline & 52 & 70.57 & 4.88 & \\
\hline & 114 & 71.04 & 25.94 & \\
\hline & 0 & 29.3 & 29.3 & \\
\hline & 5 & 4.92 & 0.001 & \\
\hline \multirow{3}{*}{ BMI } & 28 & 29.57 & 0.084 & \multirow{3}{*}{22.05} \\
\hline & 138 & 112.39 & 5.83 & \\
\hline & 10 & 33.1 & 16.12 & \\
\hline
\end{tabular}

Table 3: Chi square table for different factors.
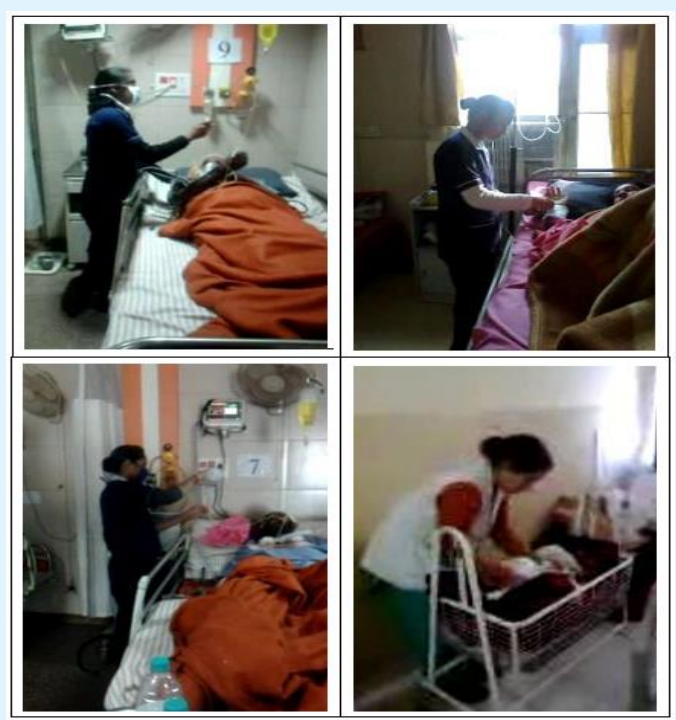

Figure 5: Nurses Working in Different postures.

\section{Conclusion}

This study reveals that the nurses that were surveyed were at high risk of musculoskeletal disorders. Awkward postures and manual patient handing in the workplace should be eliminated and hospitals should adopt ergonomics techniques and automation techniques like installation of mechanical aids such as pneumatic lifts, movable beds, wheel chairs, and/or automated handling equipment will undoubtedly decrease the risk of injuries and musculoskeletal disorders (MSDs) for nurses employed by hospitals and clinics and therefore workload can be reduced and efficiency can be increased to put the effect to organizational cost and the society as a whole. The height of the beds must be not too high and not too low. Low heights of the beds will require the nurses to bend more and if the beds are too high, the patients will experience difficulties on getting on and off the bed. The CHI square test was carried out to look into the significance of the data obtained through the surveys and 


\section{Ergonomics International Journal}

questionnaires. Majority of the nurses complained of problems in their feet and back as a result of continuous and repeated walking and bending during their hours of work. Thus the CHI square test was carried out focusing on these 2 postures as the main cause of MSDs in nurses. The calculated data at 2 degrees of freedom varies significantly when compared to the observed data. It is recommended that the workload and the work hours of the nurses be diminished and there should be a proper training of medical practices by ergonomics techniques and well defined institution layout.

\section{References}

1. http://www.thehumanfactorblog.com/2009/03/16/t he-many-definitions-of-human-factors-andergonomics/\#sthash.ocDcqB2j.dpuf

2. Table R8 (2011) Incidence rates for nonfatal occupational injuries and illnesses involving days away from work per 10,000 full-time workers by industry and selected events or exposures leading to injury or illness, private industry,

3. BLS Table R9(2010) Number of nonfatal occupational injuries and illnesses involving days away from work by occupation and selected natures of injury or illness, private industry.

4. Esterly C (2007) Strategies for an aging workforce. Presented at the Central Oregon Occupational Safety $\&$ Health Conference.

5. Safe Patient Handling and Mobility (SPHM).

6. Hignett $S$, Crumpton E, Ruszala S, Alexander P, Fray $M$, et al. (2003) Evidence-based patient handling: Systematic review. Nursing Standard 17(33): 33-36.

7. Robert Norman, Richard Wells (1998) Ergonomic Interventions for Reducing Musculoskeletal
Disorders: An Overview, Related Issues and Future Directions, University of Waterloo.

8. Jaffar N, TharimI AHB, Mohd-Kamar IF, Lop NS (2011) A Literature Review of Ergonomics Risk Factors in Construction Industry. Procedia Engineering 20: 8997.

9. Tuohy-Main K (1997) Why manual handling should be eliminated for resident and career safety. Geri action 15: 10-14.

10. DeCastro A (2004) Handle with Care: The American Nurses Association's campaign to address workrelated musculoskeletal disorders. Online Journal of Issues in Nursing 9(3): 3.

11. Interior Health (2003) MSIP: A practical guide to resident handling.

12. Nelson A, Baptiste AS (2006) Evidence-based practices for safe patient handling and movement. Online Journal of Issues in Nursing 25(6): 366-379.

13. Joint Commission (2002) Healthcare at the crossroads: Strategies for addressing the evolving nursing crisis.

14. Preventing back pain at work and home (2007) American Academy of Orthopedic Surgeons.

15. Stone PW, Clarke S, Cimiotti J, Correa-de-Araujo R (2004) Nurses' Working Conditions: Implications for Infectious Disease. Emerg Infect Dis 10(11): 19841989.

16. Susan Fralick-Ball is a licensed neuropsychologist, a registered nurse, and certified medical hypnotist. She is the founder of Maple Hypnosis and Wellness Center, Maple Gen, PA. 